\title{
Annealed Ising model with site dilution on self-similar structures
}

\author{
V. S. T. Silva, ${ }^{1}$ R. F. S. Andrade, ${ }^{1}$ and S. R. Salinas ${ }^{2}$ \\ ${ }^{1}$ Instituto de Física, Universidade Federal da Bahia, 40210-210 Salvador, Brazil \\ ${ }^{2}$ Instituto de Física, Universidade de São Paulo, Caixa Postal 66318, 05315-970 São Paulo, São Paulo, Brazil
}

(Received 10 August 2014; published 11 November 2014)

\begin{abstract}
We consider an Ising model on the triangular Apollonian network (AN), with a thermalized distribution of vacant sites. The statistical problem is formulated in a grand canonical ensemble, in terms of the temperature $T$ and a chemical potential $\mu$ associated with the concentration of active magnetic sites. We use a well-known transfer-matrix method, with a number of adaptations, to write recursion relations between successive generations of this hierarchical structure. We also investigate the analogous model on the diamond hierarchical lattice (DHL). From the numerical analysis of the recursion relations, we obtain various thermodynamic quantities. In the $\mu \rightarrow \infty$ limit, we reproduce the results for the uniform models: in the AN, the system is magnetically ordered at all temperatures, while in the DHL there is a ferromagnetic-paramagnetic transition at a finite value of $T$. Magnetic ordering, however, is shown to disappear for sufficiently large negative values of the chemical potential.
\end{abstract}

DOI: 10.1103/PhysRevE.90.052112

PACS number(s): $05.50 .+\mathrm{q}, 89.75 . \mathrm{Hc}, 64.60 . \mathrm{aq}, 75.50 . \mathrm{Lk}$

\section{INTRODUCTION}

Apollonian networks (ANs) are scaling-invariant graphs with a number of small-world properties [1,2]. Triangular Apollonian networks are generated by a recurrence procedure, which starts with a single triangle that is divided into three smaller triangles by considering a central node and linking this node to the vertices of the original triangle. This same procedure is successively repeated with all the newly formed triangles. Investigations of the behavior of statistical models on ANs, in particular spin model systems, have been reported in some recent publications [1-3]. The methods to treat these problems take advantage of the geometric scale invariance of the network. The well-known star-triangle transformation $[4,5]$ leads to exact real-space renormalization-group transformations for the simple Ising model on the standard (triangular) AN with nearest-neighbor and homogeneous interactions [2]. Suitable adaptations of the transfer matrix (TM) method have been used to treat slightly more involved problems, such as a network with generation-dependent node-node interactions and networks with nodes of distinct degrees $[1,3,6]$, and to investigate the effects of quenched disorder [7].

Previous analyses of uniform ferromagnetic Ising and Potts models on the AN indicate that there is no temperaturedependent phase transition $[1,3]$. If the coupling constants $J_{i j}$ between nodes $i$ and $j$ depend on the node degree $k$, with $J_{i j}=J_{0} /\left(k_{i} k_{j}\right)^{\alpha}$, there is a crossover value $\alpha=1$, so that the system is magnetically ordered for $\alpha<1$ and completely disordered for $\alpha>1$ [6]. The same type of ordering still remains if the model includes two nearest-neighbor antiferromagnetic interactions in each triangle in the network, but it is completely destroyed if we consider triangular plaquettes with antiferromagnetic couplings only. In a recent work, it has been numerically shown that there is no critical behavior even in a spin-glass version of the Ising model on the Apollonian network [2].

In this paper we consider the relatively simple case of a thermalized (annealed) site-diluted spin-1/2 Ising model on a triangular AN. There are a number of motivations to carry out these calculations. At the mean-field level, it is known that thermalized site dilution may change the critical behavior of the Ising model into a discontinuous phase transition $[8,9]$. We then hope that a signature of this effect may still be present in a hierarchical structure, even if we start from a situation with no phase transition. Also, it is important to point out that Apollonian networks may be used to model a large variety of soft-matter systems [10], including situations of annealed disorder [11,12], which do not require the use of the more involved methods associated with the quenched disorder of solid-state physics. The TM methodology developed in this investigation may be easily extended to treat several lattice models of physical interest.

In this work we consider two sets of degrees of freedom, orientational Ising spin variables $\left\{\sigma_{i}= \pm 1\right\}$ and site-diluted variables $\left\{\tau_{i}=0,1\right\}$ on the sites of an Apollonian network where $\tau_{i}=1$ if a site $i$ is occupied, and $\tau_{i}=0$ if a site $i$ is empty. In this model, we remark that "empty" sites as well as occupied sites can have +1 and -1 spin values. In a thermalized system, the orientational variables are treated on the same basis as the positional disorder degrees of freedom. It is then convenient to formulate the statistical problem in terms of an effective Hamiltonian in a grand-canonical ensemble, depending on temperature $T$ and a chemical potential $\mu$, which is associated with the concentration of occupied sites. It is known that this problem can be mapped onto a spin-1 Hamiltonian [10], but in this case the star-triangle transformation does not work without the inclusion of some extra multispin interaction terms. We then resort to an adaptation of the TM method, which is particularly effective for this type of problem. This TM scheme amounts to deriving a number of (nonlinear, discrete) maps for the free energy and other auxiliary variables through successive generations of the hierarchical structure. In simple situations, such as the problem of the Ising model with nearest-neighbor interactions, which do not require the addition of extra multispin interactions, the TM maps are completely equivalent to the recursion relations of the usual real-space renormalization-group $(\mathrm{RG})$ calculations. For more complex model systems, such as we investigate in the present work, the TM scheme seems more advantageous. The TM maps are derived in a straightforward way, requiring no assumptions about the form of the renormalized Hamiltonian. The only difficulty is the size of the transfer matrix and the 
number of independent matrix elements to be renormalized. Although the site-diluted model on the AN demands the iteration of ten independent mapping equations, this may be an easier task than manipulating the extra multispin interaction terms required by the application of the usual RG technique.

Thermodynamic and magnetic quantities are obtained by taking temperature or field derivatives of the free energy and require an extra number of maps for each derivative [13]. This investigation indicates that the site-diluted system on the Apollonian network is disordered for $\mu<\mu_{c}$, and orders ferromagnetically if $\mu \geqslant \mu_{c}$. This formalism reproduces all of the results for the corresponding uniform model (in the $\mu \rightarrow \infty$ limit). We have also analyzed the same site-diluted problem on a diamond hierarchical lattice (DHL) $[14,15]$, which provides a point of contact with results for a real Euclidean lattice. Indeed, it is known that the exact recursion relations for the simple DHL structure correspond to the approximate relations of the Migdal-Kadanoff bonding-moving scheme for the square lattice [14].

This article is organized as follows. In Sec. II we discuss some basic features of the annealed Ising model and the construction of the Apollonian network. Section III is devoted to the development of the transfer-matrix formalism and the derivation of the recursion maps for the AN structure. Results are discussed in Sec. IV, with emphasis on the differences between the AN and the DHL structures. Some concluding remarks are presented in Sec. V.

\section{THE DILUTED MODEL AND SELF-SIMILAR STRUCTURES}

The ferromagnetic site-diluted Ising model is given by the Hamiltonian

$$
\mathcal{H}_{g}=-J \sum_{(i, j)} \tau_{i} \tau_{j} \sigma_{i} \sigma_{j}-H \sum_{i=1}^{N_{g}} \tau_{i} \sigma_{i},
$$

where $\tau_{i}=0,1, \sigma_{i}= \pm 1$, the first sum is performed over firstneighbor pairs of sites, $J>0$, and the subscript $g$ indicates a restriction to a particular generation $g$ of the hierarchical structure. We use the label $g$ for all related quantities in the $g$ generation, such as the total number of sites (nodes) $N_{g}$, and the number of edges (bonds) $B_{g}$, of this generation. Given a configuration $\left\{\tau_{i}\right\}$, we write the total number of occupied (magnetic) sites,

$$
N_{g}^{+}=\sum_{i} \tau_{i}=N_{g} \rho_{g},
$$

where $\rho_{g}$ is the number density of occupied sites.

The hierarchical construction, which is based on the recursive substitution of a simple geometrical element by a more complex set of elements, turns out to be particularly suitable to be treated by using analytic methods that explore scaleinvariant properties, such as real-space renormalization-group $[14,15]$ and transfer-matrix techniques $[13,16]$. However, in comparison with other hierarchical structures, ANs have a much shorter history in the physics literature [17]. They can be derived in the context of a problem of filling the plane space with tangent circles, as seems to have been first proposed by the Greek geometer Apollonius of Perga. The AN is simply
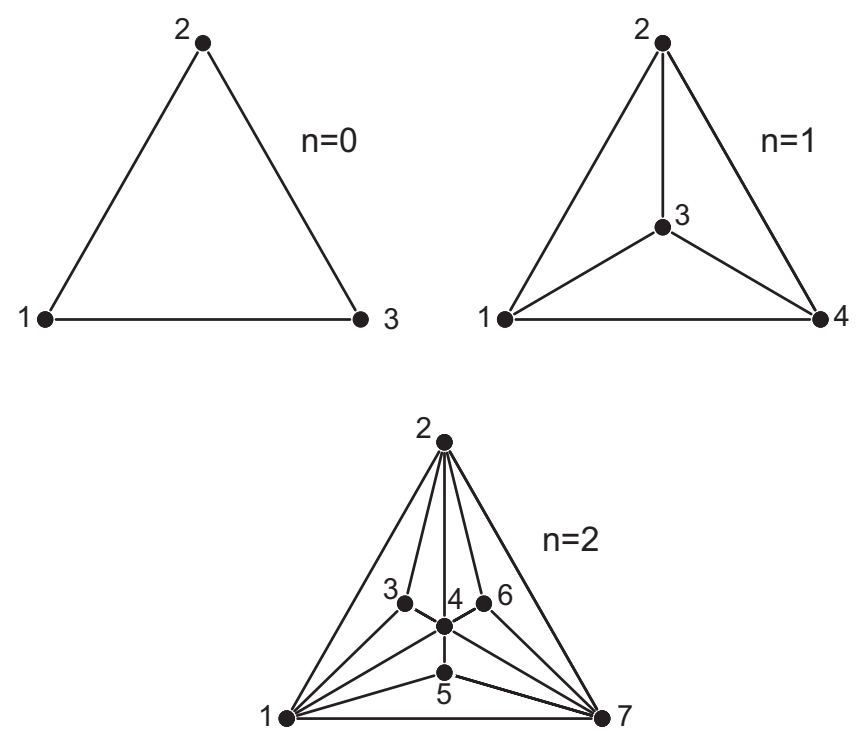

FIG. 1. Geometrical construction of the first three generations ( $g=0,1$, and 2$)$ of the AN.

related to the resulting circle pattern, since it can be defined by edges linking the centers of any pair of tangent circles. In Fig. 1 we show the first AN generations, and illustrate how we have to proceed to go from generation $g$ to generation $g+1$. In this construction, we just place a node within each existing triangle and connect this node to the three corners of the triangle.

In the AN, we have $N_{g}=\left(3^{g}+5\right) / 2$ and $B_{g}=\left(3^{g+1}+3\right) / 2$ at each generation. The inflation rule that is based on the inclusion of new nodes in the space limited by the edges, but not along the edges themselves, keeps a unit distance between the first three nodes of the initial $g=0$ generation. The largest distance between any two nodes increases with the logarithm of the total number of sites. The short distance of a given site from any other site in its neighborhood is the reason for the peculiar collective behavior of the AN as compared to Euclidian lattices and even to geometrical structures such as the diamond hierarchical lattices and Cayley trees.

\section{TM MAPS FOR ANNEALED DISORDER}

Although the TM method has been discussed in a series of articles, we believe that it is relevant to call attention to some different features associated with the treatment of annealed disorder. In this case, orientation and disorder degrees of freedom are treated at the same level. We then assume a fixed value of the number $N_{g}^{+}$of occupied sites, and write the canonical partition function

$$
Z_{g}\left(T, H, N_{g}, N_{g}^{+}\right)=\sum_{\left\{\sigma_{i}\right\}} \sum_{\left\{\tau_{i}\right\}}^{\prime} \exp \left(-\beta \mathcal{H}_{g}\right),
$$

where $\beta=1 / T, \mathcal{H}_{g}$ is given by Eq. (1), and the prime in the sum over the dilution configurations $\left\{\tau_{i}\right\}$ indicates the restriction given by Eq. (2). This restriction is removed if we introduce a chemical potential $\mu$ and change to a grand-canonical ensemble. The grand partition function is 
given by

$$
\Xi_{g}\left(T, H, N_{g}, \mu\right)=\sum_{N_{g}^{+}} Z_{g}\left(T, H, N_{g}, N_{g}^{+}\right) \exp \left(\beta \mu \sum_{i} \tau_{i}\right),
$$

which can be written in terms of unrestricted sums,

$$
\begin{aligned}
\Xi_{g}\left(T, H, N_{g}, \mu\right)= & \sum_{\left\{\sigma_{i}\right\}} \sum_{\left\{\tau_{i}\right\}} \exp \left[\beta J \sum_{(i, j)} \tau_{i} \tau_{j} \sigma_{i} \sigma_{j}\right. \\
& \left.+\beta H \sum_{i=1}^{N_{g}} \tau_{i} \sigma_{i}+\beta \mu \sum_{i=1 N_{g}} \tau_{i}\right] .
\end{aligned}
$$

In this formalism, the concentration of magnetic atoms is given by

$$
\rho_{g}=\frac{\left\langle N_{g}^{+}\right\rangle}{N_{g}}=\frac{1}{N_{g}} \frac{\partial \ln \Xi_{g}}{\partial \mu} .
$$

It is interesting to remark that the grand partition function, given by Eq. (5), is related to the partition function of a Blume-Capel (BC) model [18-21] on the same structure. This relation is obtained if (i) the three states of the variable $\sigma_{\mathrm{BC}}=+1,-1,0$ are associated with the $(1,1),(1,-1)$, and $(0, \pm 1)$ pairs of $(\tau, \sigma)$ variables; (ii) the crystalline field, which is usually represented by an interaction parameter $D$ in the BC Hamiltonian, is associated with the chemical potential $\mu$, and takes into account the double degeneracy of the $\sigma_{\mathrm{BC}}=0$ state. In the present work, however, it is not interesting to resort to this equivalence.

Let us briefly sketch the TM formalism. Given a twostate system Hamiltonian $\mathcal{H}_{0}=-J \tau_{1} \tau_{2} \sigma_{1} \sigma_{2}$, we begin the application of the formalism by writing the matrix

$$
M_{0}=\left(\begin{array}{cccc}
a_{0} & b_{0} & d_{0} & d_{0} \\
b_{0} & e_{0} & f_{0} & f_{0} \\
d_{0} & f_{0} & c_{0} & c_{0} \\
d_{0} & f_{0} & c_{0} & c_{0}
\end{array}\right) .
$$

Any row or column label (say $i=1,2,3,4$ ) corresponds to the pairs of values $(1,1),(1,-1),(0,1)$, and $(0,-1)$, in the expression of the Hamiltonian $\mathcal{H}_{0}\left(\tau_{i}, \sigma_{i}\right)$. Although the Boltzmann weights expressed by the matrix elements of $M_{0}$ can take only three different values $(\beta J,-\beta J$, and 0$)$, we write $M_{0}$ with six distinct entries. The main reason is that, for larger values of $g$, nonzero values of $\mu$ and $H$ break this large degeneracy and require a more complex structure of elements, as displayed by Eq. (7). The matrix $M_{0}$ contains all Boltzmann weights depending on different two-spin configurations of the two outer sites limiting the geometric substrate. The TM method amounts to constructing similar matrices $M_{g}$, the elements of which depend on the configurations of the outer sites only, and which consists of partial traces over all contributions of the configurations of the internal sites.

We point out that, since both $\mu$ and $H$ are associated with each site, the field contributions are taken only once as we perform the sums in Eq. (5) through successive generations. It should be emphasized that the system at generation $g$ is formed by sharing a subset of sites belonging to the $g-1$ generation. Some of these sites play no role in the construction of generation $g+1$, and will be called internal sites of $g$. The sites that take part in the construction of the new $g+1$ structure are called external (or outer) sites of generation $g$. The strategy to account for the field action within the TM formalism consists in writing $M_{0}$ in a field-free situation and, as we go from generation $g-1$ to $g$, letting the fields act only on those external sites of $g-1$ that become internal sites of generation $g$. For instance, the fields are applied in site $i=4$ at the generation $g=2$, as illustrated in Fig. 1 .

The AN triangular structure requires the consideration of nonsquare matrices. For $g=1$, we define a matrix $B_{1}$ given by the elements

$$
\left(B_{1}\right)_{i, j k}=\left(M_{0}\right)_{i, j}\left(M_{0}\right)_{j, k}\left(M_{0}\right)_{i, k},
$$

with $i, j, k=1,2,3,4$. The square matrix $\left(M_{1}\right)_{i, k}$ is obtained by taking the trace of $B_{1}$ over the index $j$. For $g \geqslant 1$, the recurrence maps for the matrix elements of $B_{g+1}$ and $M_{g+1}$ are given by

$$
\begin{aligned}
\left(B_{g+1}\right)_{i, j k}= & \sum_{\ell}\left(B_{g}\right)_{i, \ell k}\left(B_{g}\right)_{i, \ell j}\left(B_{g}^{T}\right)_{\ell k, j} \\
& \times \exp \left\{\beta\left[\left(h \sigma_{\ell} \tau_{\ell}+\mu \tau_{\ell}\right) / 2\right]\right\}
\end{aligned}
$$

and

$$
\left(M_{g+1}\right)_{i, j}=\sum_{k}\left(B_{g+1}\right)_{i, j k}
$$

The matrices $M_{g}$ have the same structures of matrix elements as defined by Eq. (7). However, the recurrence relations cannot be expressed in simple form by matrix operations. Since the matrix $M_{g}$ is not needed for the definition of $B_{g+1}$, it is more convenient to iterate the matrix elements of matrices $B_{g}$, which are given by the structural form

$$
B_{g}=\left(\begin{array}{llllllllllllllll}
a_{g} & b_{g} & c_{g} & c_{g} & b_{g} & g_{g} & e_{g} & e_{g} & c_{g} & e_{g} & f_{g} & f_{g} & c_{g} & e_{g} & f_{g} & f_{g} \\
b_{g} & g_{g} & e_{g} & e_{g} & g_{g} & d_{g} & h_{g} & h_{g} & e_{g} & h_{g} & i_{g} & i_{g} & e_{g} & h_{g} & i_{g} & i_{g} \\
c_{g} & e_{g} & f_{g} & f_{g} & e_{g} & h_{g} & i_{g} & i_{g} & f_{g} & i_{g} & j_{g} & j_{g} & f_{g} & i_{g} & j_{g} & j_{g} \\
c_{g} & e_{g} & f_{g} & f_{g} & e_{g} & h_{g} & i_{g} & i_{g} & f_{g} & i_{g} & j_{g} & j_{g} & f_{g} & i_{g} & j_{g} & j_{g}
\end{array}\right) .
$$

We remark that there are ten distinct matrix elements. Each one of these elements obeys a recursion relation that connects the value in generation $g+1$ with the values of all the elements belonging to generation $g$. We now use a transformation of 
variables, define a free energy given by

$$
\phi_{g}(T, H, \mu)=\Phi_{g}\left(T, H, \mu, N_{g}\right) / N_{g}=-\frac{T}{N_{g}} \ln \left(a_{g}\right),
$$

as well as nine other variables that will be globally indicated by $\bar{x}_{g}=x_{g} / a_{g}$. In Appendix A, we list the complete set of ten recurrence maps that are required to obtain the results to be discussed in the following section. In Appendix B we list the corresponding maps for the diluted model system on the DHL structure.

\section{THERMODYNAMIC BEHAVIOR}

The numerical iteration of the maps in the Appendixes produces a series of results in terms of the parameters $T$, $H$, and $\mu$. For any set of these parameters, this iterative procedure produces values that rapidly converge to a fixed value. Thus, the maps are iterated until a generation $g^{*}$ so that, for numerical variables with 16 significant digits, the free energy $\phi_{g}$ becomes a constant for $g \geqslant g^{*}$. The value of $g^{*}$ depends on the particular parameters, but the convergence is usually achieved for $g<50$. The thermodynamic properties expressed in terms of derivatives of the free energy can be obtained either by numerically differentiating the $\phi_{g}$ map, for $g \geqslant g^{*}$, or by iterating a larger set of maps for the derivatives of $\phi_{g+1}$ and $\bar{x}_{g+1}$ as functions of $\phi_{g}$ and $\bar{x}_{g}$ and their derivatives. The results of these calculations can be better appreciated by showing some graphs, which were obtained by setting $J=1$.

In Fig. 2 we draw some graphs of the specific heat $\mathcal{C}$ as a function of temperature $T$, for different values of the chemical potential $\mu$, all of which display characteristic smooth Schottky profiles. A qualitative inspection of these graphs indicates that, independently of the value of $\mu$, the specific heat is always smooth for $T>0$. This might lead to the conclusion that qualitative aspects of the thermodynamic behavior are completely unaffected by the chemical potential. This is not the case, as we illustrate in Fig. 3, by drawing graphs of $\rho$ as function of $T$ for several values of $\mu$. These graphs point out the existence of a peculiar behavior at very low temperatures, with $\rho(T \rightarrow 0) \rightarrow 1$ for $\mu>-3$,

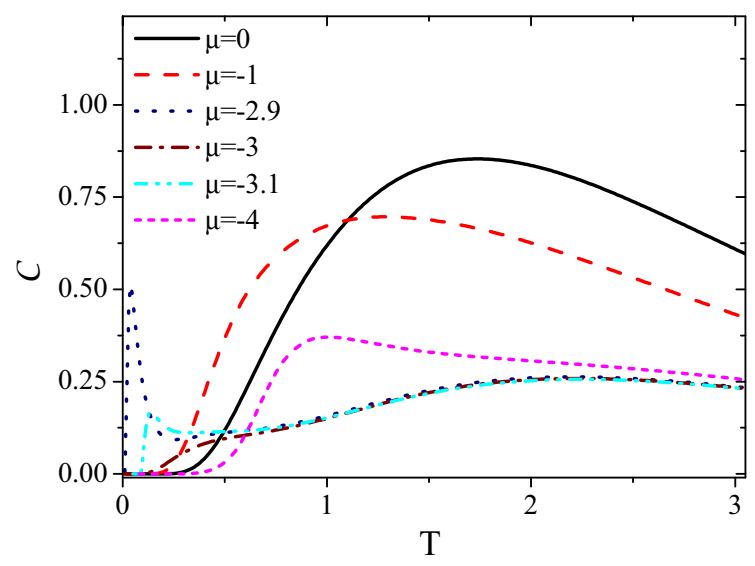

FIG. 2. (Color online) Specific heat $\mathcal{C}$ of the diluted system on the AN as a function of temperature $T$ for various values of the chemical potential $\mu$.

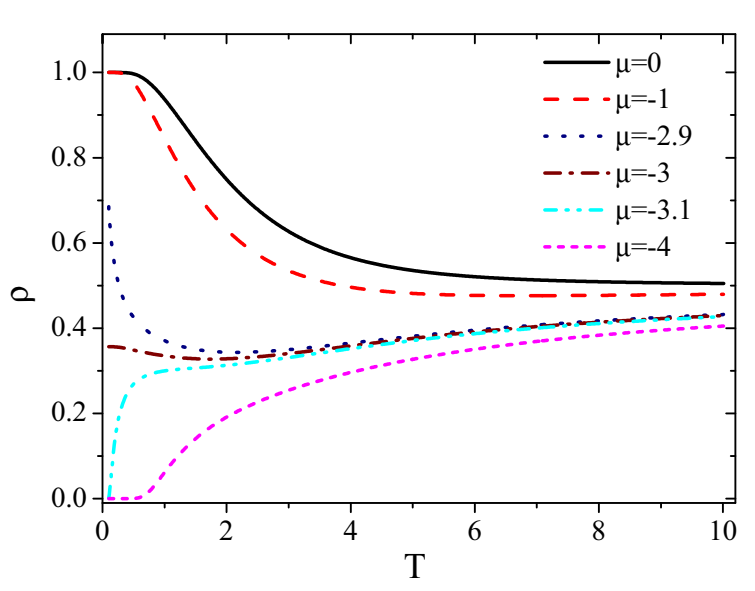

FIG. 3. (Color online) Concentration $\rho$ of the magnetic sites of the dilute model on the AN as a function of temperature for various values of chemical potential $\mu$. At zero temperature, there is a discontinuous behavior of $\rho$ as the chemical potential changes through the characteristic value $\mu=-3$.

$\rho(T \rightarrow 0) \rightarrow 0$ for $\mu<-3$, and $\rho(T \rightarrow 0)=0.35663 \ldots$ for $\mu=-3$. Strictly speaking, this nonanalytic behavior does not characterize a phase transition at a finite temperature, but it does suggest a first-order transition at $T=0$ and $\mu=-3$.

The existence of a singular behavior can also be observed in the low-temperature dependence of the entropy with respect to the chemical potential $\mu$. This can be seen in Fig. 4, which indicates the existence of a residual entropy for $\mu \leqslant-3$. It is interesting to remark that the same limiting values, $s(T=$ $0 ; H=0)=0$, for $\mu>-3$, and $s(T=0 ; H=0)=\ln 2$, for $\mu<-3$, are also obtained in the exact calculations for a one-dimensional version of this diluted Ising model. In these lattice models, at zero temperature, and sufficiently large and negative values of the chemical potential, there is an entropic effect associated with the random orientation $\left(\sigma_{i}= \pm 1\right)$ of the magnetically nonactive sites. At zero temperature and $\mu=-3$, however, the residual entropy on the AN reaches a larger value, $s(T=0 ; H=0 ;=3)=0.8432 \ldots$. This value can be

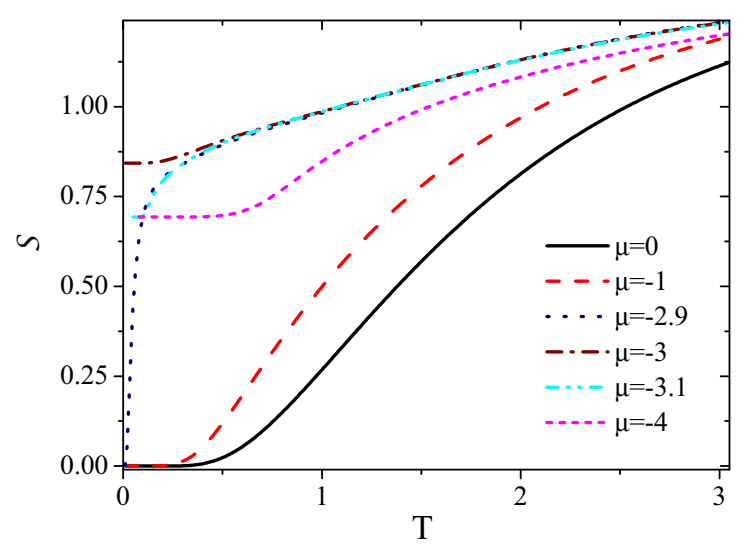

FIG. 4. (Color online) Entropy $s$ on the AN lattice as a function of temperature for various values of chemical potential $\mu$. There is a residual entropy for $\mu \leqslant-3$. At the critical value $\mu=-3$, the residual entropy is $s(T=0)=0.8432 \ldots$. This value changes to $s(T=0)=\ln 2$ for $\mu<-3$. 


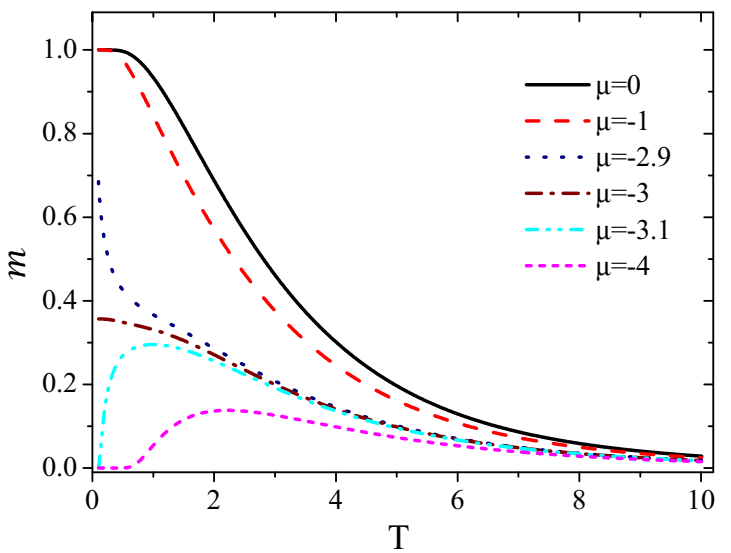

FIG. 5. (Color online) Magnetization in zero field on the AN as a function of temperature for various values of the chemical potential $\mu$. As in Fig. 3, at zero temperature, there is a discontinuous behavior of the magnetization at $\mu=-3$.

analytically derived by considering only the dominating terms in the maps in Appendix A when $T \rightarrow 0$ and $\mu=-3$. In this case, there are several configurations with $\tau=1$ that have the same Boltzmann weights as the the usual doubly degenerate configurations $\sigma= \pm 1$ and $\tau=0$, which are responsible for the residual entropy $\ln 2$. For instance, in the first generation, it is easily seen that the dominant terms are those with all sites in the $(\sigma=1, \tau=1)$ state and all external sites in the $(\sigma=1, \tau=1)$ state and the central site in the $(\sigma= \pm 1, \tau=0)$ state. In the subsequent generations, these configurations occur recurrently, while new configurations where the three external and the central sites have exactly the same configurations as for $g=1$ emerge in each new generation. As a result, not only is the residual entropy larger, but also the density of sites with magnetic properties (i.e., $\tau=1$ ) is nonzero.

The observed singular behavior of the entropy $s$ and the density $\rho$ is also confirmed by the graphs of the spontaneous magnetization, as we illustrate in Fig. 5, for different values of $\mu$.

It is interesting to remark that the spontaneous magnetization $m=-\partial \phi / \partial H$ does not become identically zero at a well-defined temperature value. The graphs of $m$, for $\mu>-3$, are similar to those obtained for the uniform, nondiluted, ferromagnetic Ising model, $m(T=0)=1$, while $m(T \rightarrow \infty) \rightarrow 0$ exponentially. The $T=0$ limit persists for
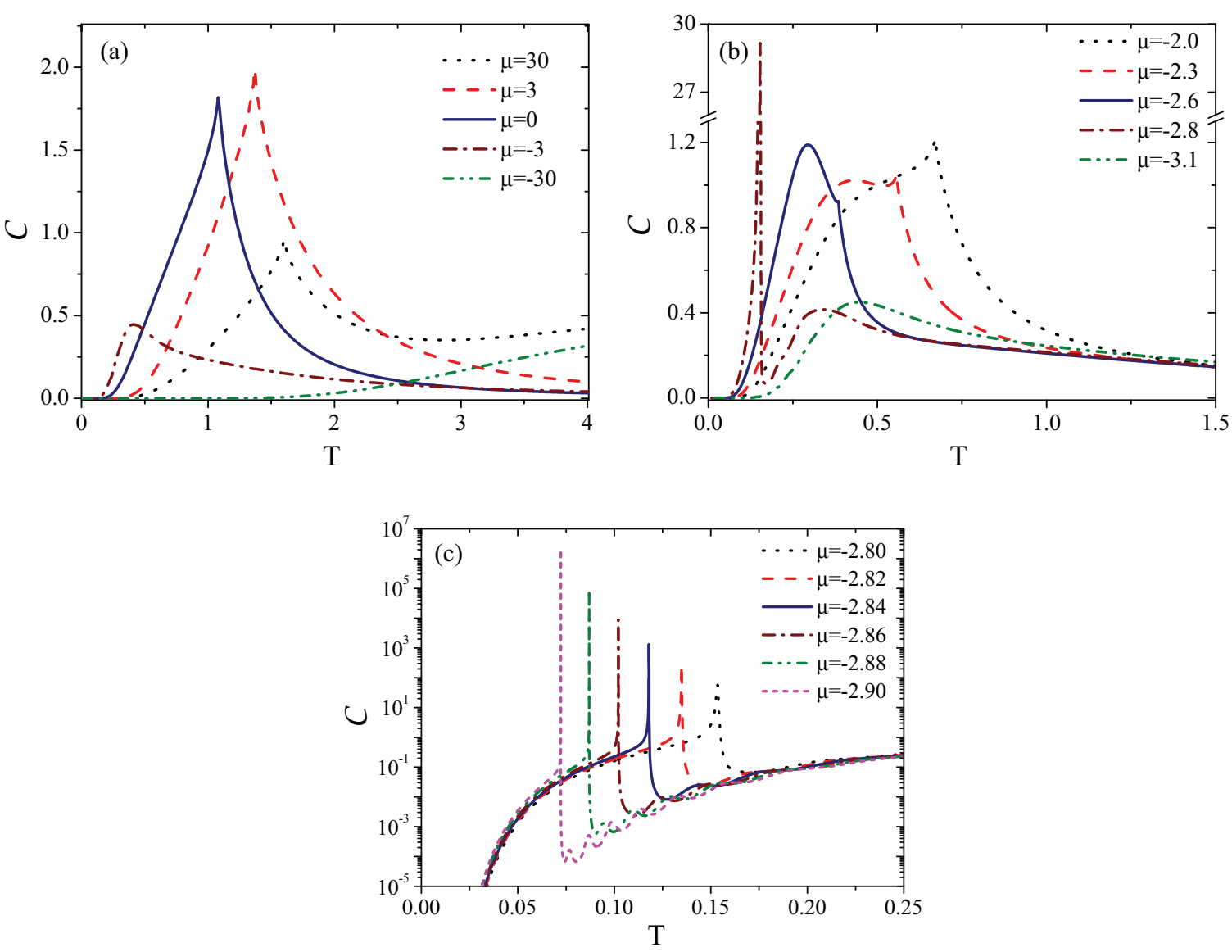

FIG. 6. (Color online) Specific heat $\mathcal{C}$ of the diluted model on the DHL as a function of temperature for several ranges of values of the chemical potential $\mu$. (a) explores large intervals of $\mu$, illustrating phase transitions at $T_{c}(\mu)$ indicated by cusps for $\mu=30,3$, and 0 . No cusp appears for $\mu \leqslant-3$. (b) illustrates patterns consisting of cusps with a broad maximum superposed for $\mu=-2.0,-2.3$, and -2.6 . At $\mu=-2.8$ the cusp to the left of the broad maximum reaches a very large value $\simeq 29.15$. (c) shows the huge increase of $\mathcal{C}\left(T_{c}\right)$ as $\mu$ decreases, which is related to the presence of a tricritical point; $\mu=-2.95$ is the lowest value of $\mu$ where the cusp can be well characterized, as $\mathcal{C}\left(T_{c}\right) \sim 10^{13}$. 
$\mu \gtrsim-3$, as observed for $\mu=-2.9$. On the other hand, $m(T=0)=0.35663 \ldots$ for $\mu=-3$, whereas $m(T=0)=0$ for $\mu<-3$.

If we restrict the calculations to the $T \rightarrow 0$ limit, the highly nonlinear maps in Appendix A can be simplified by excluding all but the necessary dominant terms. In this case, it is possible to derive analytic expressions for $\phi$ and its derivatives. This tedious although straightforward procedure leads to the confirmation of the reported values for the residual entropy, concentration, and magnetization at $\mu=-3$. These calculations also lead to asymptotic expressions for the entropy and magnetization at low temperatures $T \ll 1$.

We now turn to the behavior of the site-diluted model on the diamond hierarchical lattice, which comes from the iteration of the recursion relations that are explicitly written in Appendix B. If we consider the simple ferromagnetic Ising model, it is known that the exact recursion relations for the DHL correspond to the approximate relations for a Euclidian square lattice, as obtained by the application of the Migdal-Kadanoff bond-moving scheme. We then use the results for the DHL structure, which might be closer to a real square lattice, to provide a basis of comparison with the findings for the Apollonian structure.

In Fig. 6, we illustrate the behavior of the specific heat $\mathcal{C}$ versus temperature $T$ for several ranges of values of $\mu$. The three panels in this figure indicate distinct features of the behavior of $\mathcal{C}$ as compared to the analogous model on the AN. If we let $\mu \rightarrow \infty$, we reproduce the uniform (undiluted) model, which is characterized by the presence of a cusp singularity at $T_{c}$, the critical temperature of a second-order ferromagnetic-paramagnetic phase transition. These global features are shown in Fig. 6(a). The cusp that is associated with the second-order phase transition is observed in this figure for $\mu=0,3$, and 30 . For $\mu \leqslant-3$, the specific heat displays just a broad (Schottky-type) maximum.

In Fig. 6(b), we display the typical behavior of $\mathcal{C}$ in the interval $-2.8 \leqslant \mu \leqslant-2.0$. In this range of values of $\mu$, besides the characteristic cusp associated with the secondorder transition, we note the emergence of a broad (Schottky) maximum, whose relative position with respect to the cusp changes with $\mu$. As we mentioned in the last paragraph, this broad maximum is still present for $\mu<-3$.

In Fig. 6(c), we consider a very small interval, $-2.90 \leqslant$ $\mu \leqslant-2.80$, within which we observe important changes in the critical behavior. In this interval, as $\mu$ decreases, the height of the cusp of the specific heat, which we call $\mathcal{C}\left(T_{c}\right)$, increases very sharply. We were able to extend these calculations beyond the values in the graphs, and followed the behavior of the specific heat down to $\mu=-2.95$, with a cusp as large as $10^{13}$. The broad maximum survives for $\mu<-3$, but the values of the critical temperature are very small. Due to the quick divergence of the Boltzmann weights, we have numerical difficulties in analyzing the maps listed in the Appendixes.

In the DHL, the sharp increase of $\mathcal{C}(T)$ is related to the presence of a tricritical point. A detailed analysis of the Migdal-Kadanoff RG structure of fixed points and flow lines of the spin-1 Blume-Emery-Griffiths (BEG) model in two and three dimensions has been reported by Kaufman et al. [22]. This Migdal-Kadanoff scheme on the square lattice leads to exact recursion relations for a simple "necklace"

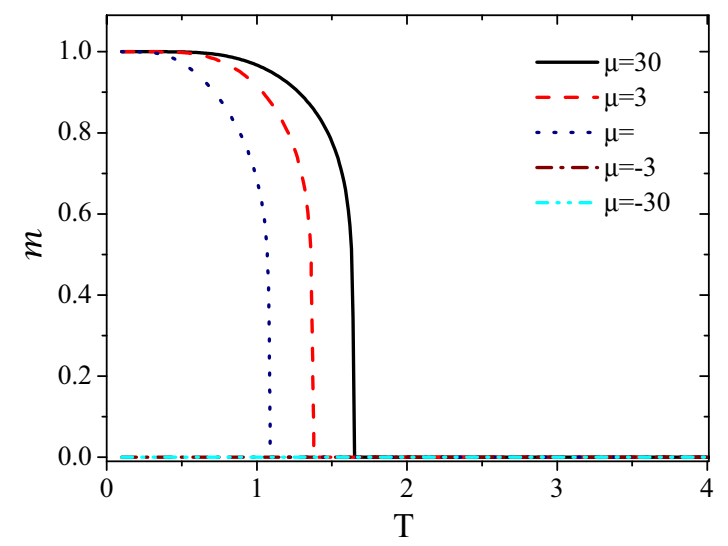

FIG. 7. (Color online) Spontaneous magnetization $m$ on the DHL as a function of temperature for various values of the chemical potential $\mu$. Note that $m \rightarrow 0$ at $T=T_{c}(\mu)$ for $\mu>-3$, and that $m \equiv 0$ at all temperatures for $\mu<-3$.

hierarchical structure, which is similar (although not identical) to the diamond hierarchical lattice that we have considered in the present paper. In view of the mapping of the annealed disordered problem into a BEG spin-1 Hamiltonian, it is then clear that we do have a tricritical point in the phase diagram of the Ising diluted system on the diamond hierarchical lattice, in terms of temperature $T$ and chemical potential $\mu$.

In Fig. 7, we illustrate the behavior of the spontaneous magnetization $m$ as function of temperature for different values of $\mu$. For $\mu>-3$, there is a non-zero spontaneous magnetization at low temperatures, which vanishes continuously as $T \rightarrow T_{c}$. For $\mu \leqslant-3$, the spontaneous magnetization $m$ vanishes identically for all values of $T$.

The values of $T_{c}$ were independently confirmed by calculations of the correlation length $\xi$ and the susceptibility $\chi$. This agreement between independent calculations supports the validity of the adaptation of the transfer matrix method to the annealed systems. We are then confident to point out the differences between the peculiar behavior of the annealed system on the Apollonian network as compared to the results for the diamond hierarchical lattice.

\section{v. CONCLUSIONS}

We have developed a suitable adaptation of the transfermatrix method to write exact recursion relations and investigate the thermodynamic behavior of a simple ferromagnetic Ising model in the presence of annealed site dilution on a triangular Apollonian network. In order to provide a basis of comparison, we have also analyzed the same spin system on a diamond hierarchical lattice, whose recursion relations are known to be identical to the approximate results obtained from an application of the Migdal-Kadanoff bonding-moving scheme to a square lattice. The problem is formulated in a grand ensemble, in terms of the temperature $T$ and a chemical potential $\mu$ associated with the concentration $\rho$ of active sites.

In the Apollonian network, although there is no phase transition at finite temperatures, there is a peculiar lowtemperature behavior in terms of the chemical potential. We show that $\rho(T \rightarrow 0) \rightarrow 1$ for $\mu>-3, \rho(T \rightarrow 0) \rightarrow 0$ for 
$\mu<-3$, and $\rho(T \rightarrow 0)=0.35663 \ldots$ for $\mu=-3$, which indicates a first-order transition at $T=0$ and $\mu=-3$. The spontaneous magnetization $m$ displays a similar peculiar behavior. For $\mu>-3$, we have $m(T=0)=1$, which is the typical result for the uniform Ising ferromagnet. Also, $m(T) \neq$ 0 for all finite temperatures, and vanishes exponentially for $T \rightarrow \infty$. On the other hand, $m(T=0)=0$ for $\mu<-3$. At $T=0$ and $\mu=-3, m$ and $\rho$ reach the same value, $m(T=$ $0)=\rho(T=0)=0.35663 \ldots$, which reflects the effect of the dilution.

We have analyzed the behavior of the zero-field entropy of the annealed system on the Apollonian network. For $\mu>$ -3 , there is no residual entropy. For $\mu<-3$, we find the residual entropy $s(T=0, H=0)=\ln 2$, which can be easily understood in terms of a random occupation of the sites. At $\mu=-3$, however, the residual entropy reaches a larger value, $s(T=0, H=0, \mu=-3)=0.8432 \ldots$.

The thermodynamic behavior of the annealed system on the DHL also presents interesting features. We paid particular attention to the behavior of the specific heat as a function of temperature for several values of $\mu$. In the undiluted case, it presents a cusp singularity at $T_{c}$, the temperature of a second-order phase transition. In the diluted case, we find no critical behavior for $\mu \leqslant-3$. However, the same kind of cusp singularity characterizes the interval $\mu>-3$. Our calculations have indicated a sharp increase of $\mathcal{C}\left(T_{c}\right)$ when $\mu \simeq-2.95$, which is consistent with the presence of a tricritical point, as has been discussed in previous studies. When $\mu \rightarrow \infty$, the results of the undiluted model are reproduced. We have also found that a residual entropy is found in the zero-temperature limit when $\mu<-3$. The calculation of the spontaneous magnetization as a function of temperature, for different values of $\mu$, provides additional information on the critical line.

\section{ACKNOWLEDGMENTS}

The authors acknowledge the financial support of Brazilian agencies CNPq, CAPES, and FAPESB. R.F.S.A. also thanks the Instituto Nacional de Ciência e Tecnologia para Sistemas Complexos (INCT-SC) for support.

\section{APPENDIX A}

The recurrence maps for the ten distinct matrix elements shown in Eq. (11) are rewritten in terms of the potential $\phi_{g}$ defined by Eq. (12) and rescaled variables given by the ratio of the other matrix elements by $a_{g}$ according to the following definitions: $r_{g}=b_{g} / a_{g}, s_{g}=c_{g} / a_{g}, p_{g}=d_{g} / a_{g}, t_{g}=e_{g} / a_{g}$, $u_{g}=f_{g} / a_{g}, q_{g}=g_{g} / a_{g}, w_{g}=h_{g} / a_{g}, x_{g}=i_{g} / a_{g}, v_{g}=j_{g} / a_{g}$. We also define $y=\exp (\beta \mu / 2)$ and $z=\exp (\beta h)$, and recall that $N_{g}=\left(3^{g}+5\right) / 2$.

$$
\begin{aligned}
\phi_{g+1} & =3 \phi_{g} \frac{N_{g}}{N_{g+1}}-\frac{T}{N_{g+1}} \ln \left[2 s_{g}^{3}+\left(r_{g}^{3} y\right) / z+y z\right], \\
r_{g+1} & =\frac{2 s_{g} t_{g}^{2}+\left(r_{g} q_{g}^{2} y\right) / z+r_{g}^{2} y z}{2 s_{g}^{3}+\left(r_{g}^{3} y\right) / z+y z},
\end{aligned}
$$

$$
\begin{aligned}
& s_{g+1}=\frac{2 s_{g} u_{g}^{2}+\left(r_{g} t_{g}^{2} y\right) / z+s_{g}^{2} y z}{2 s_{g}^{3}+\left(r_{g}^{3} y\right) / z+y z}, \\
& q_{g+1}=\frac{2 t_{g}^{2} w_{g}+\left(p_{g} q_{g}^{2} y\right) / z+r_{g}^{2} q_{g} y z}{2 s_{g}^{3}+\left(r_{g}^{3} y\right) / z+y z}, \\
& t_{g+1}=\frac{2 t_{g} u_{g} x_{g}+\left(t_{g} q_{g} w_{g} y\right) / z+r_{g} s_{g} t_{g} y z}{2 s_{g}^{3}+\left(r_{g}^{3} y\right) / z+y z}, \\
& u_{g+1}=\frac{2 u_{g}^{2} v_{g}+\left(t_{g}^{2} x_{g} y\right) / z+s_{g}^{2} u_{g} y z}{2 s_{g}^{3}+\left(r_{g}^{3} y\right) / z+y z}, \\
& p_{g+1}=\frac{2 w_{g}^{3}+\left(p_{g}^{3} y\right) / z+q_{g}^{3} y z}{2 s_{g}^{3}+\left(r_{g}^{3} y\right) / z+y z}, \\
& w_{g+1}=\frac{2 w_{g} x_{g}^{2}+\left(p_{g} w_{g}^{2} y\right) / z+t_{g}^{2} q_{g} y z}{2 s_{g}^{3}+\left(r_{g}^{3} y\right) / z+y z}, \\
& x_{g+1}=\frac{2 x_{g}^{2} v_{g}+\left(w_{g}^{2} x_{g} y\right) / z+t_{g}^{2} u_{g} y z}{2 s_{g}^{3}+\left(r_{g}^{3} y\right) / z+y z}, \\
& v_{g+1}=\frac{2 v_{g}^{3}+\left(x_{g}^{3} y\right) / z+u_{g}^{3} y z}{2 s_{g}^{3}+\left(r_{g}^{3} y\right) / z+y z} .
\end{aligned}
$$

\section{APPENDIX B}

The TM formalism for the diluted model on the DHL results in a set of six maps for distinct elements of a $4 \times 4$ matrix with the same structure as in Eq. (7). As in the case of the $\mathrm{AN}$, they are rewritten in terms of the potential $\phi_{g}$ defined by Eq. (12) and rescaled variables given by the ratio of the other matrix elements to $a_{g}$ according to the following definitions: $r_{g}=b_{g} / a_{g}, s_{g}=e_{g} / a_{g}, t_{g}=d_{g} / a_{g}, x_{g}=c_{g} / a_{g}, v_{g}=f_{g} / a_{g}$. As in Appendix A, we define $y=\exp (\beta \mu / 2)$ and $z=\exp (\beta h)$, but now $N_{g}=2\left(4^{g}+2\right) / 3$.

$$
\phi_{g+1}=4 \phi_{g} \frac{N_{g}}{N_{g+1}}-\frac{T}{N_{g+1}} \ln \left\{\left[r_{g}^{2} y+z\left(2 t_{g}^{2}+y z\right)\right]^{2} / z^{2}\right\},
$$

$$
\begin{aligned}
& r_{g+1}=\frac{\left[2 t_{g} v_{g} z+r_{g} y\left(s_{g}+z^{2}\right)\right]^{2}}{\left[r_{g}^{2} y+z\left(2 t_{g}^{2}+y z\right)\right]^{2}}, \\
& s_{g+1}=\frac{\left[s_{g}^{2} y+z\left(2 v_{g}^{2}+r_{g}^{2} y z\right)\right]^{2}}{\left[r_{g}^{2} y+z\left(2 t_{g}^{2}+y z\right)\right]^{2}}, \\
& t_{g+1}=\frac{\left[r_{g} v_{g} y+t_{g} z\left(2 x_{g}+y z\right)\right]^{2}}{\left[r_{g}^{2} y+z\left(2 t_{g}^{2}+y z\right)\right]^{2}}, \\
& x_{g+1}=\frac{\left[v_{g}^{2} y+z\left(2 x_{g}^{2}+t_{g}^{2} y z\right)\right]^{2}}{\left[r_{g}^{2} y+z\left(2 t_{g}^{2}+y z\right)\right]^{2}}, \\
& v_{g+1}=\frac{\left[s_{g} v_{g} y+z\left(2 v_{g} x_{g}+r_{g} t_{g} y z\right)\right]^{2}}{\left[r_{g}^{2} y+z\left(2 t_{g}^{2}+y z\right)\right]^{2}} .
\end{aligned}
$$


[1] R. F. S. Andrade and H. J. Herrmann, Phys. Rev. E 71, 056131 (2005).

[2] C. N. Kaplan, M. Hinczewski, and A. N. Berker, Phys. Rev. E 79, 061120 (2009).

[3] N. A. M. Araújo, R. F. S. Andrade, and H. J. Herrmann, Phys. Rev. E 82, 046109 (2010).

[4] I. Syozi, in Phase Transitions and Critical Phenomena (Academic Press, London, 1972), Vol. 1, p. 270.

[5] H. J. Hilhorst, M. Schick, and J. M. J. van Leeuwen, Phys. Rev. B 19, 2749 (1979).

[6] R. F. S. Andrade, J. S. Andrade, Jr., and H. J. Herrmann, Phys. Rev. E 79, 036105 (2009).

[7] D. O. C. Santos, E. Nogueira, Jr., and R. F. S. Andrade, Phys. Rev. B 73, 174202 (2006).

[8] A. A. Lushnikov, Phys. Lett. A 40, 303 (1972).

[9] M. Wortis, Phys. Lett. A 47, 445 (1974).

[10] A. M. Figueiredo-Neto and S. R. Salinas, The Physics of Lyotropic Liquid Crystals: Phase Transitions and Structural Properties (Oxford Science Publications, Oxford, 2005).
[11] E. doCarmo, D. B. Liarte, and S. R. Salinas, Phys. Rev. E 81, 062701 (2010)

[12] E. doCarmo, A. P. Vieira, and S. R. Salinas, Phys. Rev. E 83, 011701 (2011).

[13] R. F. S. Andrade, Phys. Rev. B 48, 16095 (1993).

[14] A. N. Berker and S. Ostlund, J. Phys. C 12, 4961 (1979).

[15] M. Kaufman and R. B. Griffiths, Phys. Rev. B 24, 496 (1981).

[16] R. F. S. Andrade, Phys. Rev. E 59, 150 (1999).

[17] J. S. Andrade, Jr., H. J. Herrmann, R. F. S. Andrade, and L. R. da Silva, Phys. Rev. Lett. 94, 018702 (2005).

[18] M. Blume, Phys. Rev. 141, 517 (1966).

[19] H. W. Capel, Physica (Amsterdam) 32, 966 (1966).

[20] M. Blume, V. J. Emery, and R. B. Griffiths, Phys. Rev. A 4, 1071 (1971).

[21] A. N. Berker and M. Wortis, Phys. Rev. B 14, 4946 (1976).

[22] M. Kaufman, R. B. Griffiths, J. M. Yeomans, and M. E. Fisher, Phys. Rev. B 23, 3448 (1981). 